\title{
Early observation of modal parameter changes by an enhanced frequency evaluation algorithm
}

\author{
I.C. Mituletu, G.R. Gillich, M. Tufoi, N. Gillich \\ Universitatea "Eftimie Murgu” din Resita, Department of Mechanical Engineering, 320085 Resita, Romania
}

\begin{abstract}
By monitoring the vibration response of mechanical or civil engineering systems, the modal parameter changes due to materials or geometrical properties can be observed. Fine frequency shift identification is decisive to determine particularly small changes and thereby small cracks or damages. This paper firstly presents some existing methods to accurately find the frequency values. All these methods show non-linear, relevant errors in interpretation. Afterward a simple method is proposed, consisting in iterative decreasing of the number of samples from the original signal, performing a standard frequency analysis for each truncated signal and finally overlapping of achieved spectra. Thus, the resulted spectrum has a much fine resolution due to the important addition of spectral lines. In this way a more accurate identification of frequencies is possible. In fact, the relevant frequency is accomplished under a lobe shape. In the peak position of this lobe the best frequency value can be clearly identified. With the help of these more accurate frequency values, modal analysis becomes more reliable and efficient.
\end{abstract}

\section{Introduction}

Methods which based on the changes of the structural modal parameters are commonly involved to establish the global health of a mechanical or civil engineering structure and to assess damages. The methods based on frequency changes are easiest applied, but problems occur because small damage produces small changes in the natural frequencies. Thus, observe the changes in the frequency spectrum are hard to be found [1]. In standard frequency evaluation, the problem is that frequencies are indicated on spectral lines, whose location directly dependents on the analyzed signal length. As a consequence, for small damages, the frequency changes are not indicated in the spectrum until the peak-amplitude does not move to the inferior spectral line. For early damage observation, it becomes necessary to involve advanced algorithms, providing denser spectral lines, with direct consequence increasing the frequency evaluation accuracy. In standard frequency evaluation often false results are signalized. To understand why this happens, we review some notions of mathematics and signal analysis.

By applying the Fourier Transform (FT) to an infinite long signal in time, an infinitely small frequency resolution is achieved. Thus, the frequency is precisely identified. For a finite-time length signal, the resolution becomes relevant, depending on the time length. To calculate the frequency content for discrete signal $x$, the Discrete Fourier Transform (DFT) can be used. It provides, in a simple way, information about the frequency spectrum of the signal, by turning $N$ samples of the signal from the time domain in $N$ complex values in the frequency domain. Its mathematical expression is:

$X\left(f_{k}\right)=\sum_{n=0}^{N-1} x\left(t_{n}\right) e^{-j 2 \pi f_{k} t_{n}}=\sum_{n=0}^{N-1} x\left(t_{n}\right) e^{-j 2 \pi \frac{k}{N-1} n}$

with $k=0,1,2, \ldots N-1$.

In Eq. (1) we denoted $x\left(t_{n}\right)$ the instantaneous input signal amplitude at time $t_{n}=n T, T$ the sampling period, $X\left(f_{k}\right)$ the spectrum of $x$ at frequency $f_{k}$ and $N$ the number of time samples. The signal time length results in $t_{N-1}$.

The Power Spectrum Density (PSD) is a positive real function of a frequency variable. While the Power Spectrum (PS) calculates the area under the signal plot using the discrete FT, the power spectrum density assigns units of power to each unit of frequency and so, enhances periodicities.

Appling the PSD to a sequence representing a sinusoidal signal, one can distinguish between two cases. First, if the sequence contains an integer number of periods, as depicted in figure 1a, the frequency is read precisely. It is exemplified in figure 1c for a signal whit a created frequency of $4 \mathrm{~Hz}$, analyzed in the time interval of $1 \mathrm{~s}$. The PSD, represented by a red line, indicates the correct frequency.

In the second case, the signal sequence does not contain an integer number of periods. This is illustrated in 
figure $1 \mathrm{~b}$. For this signal, also of $4 \mathrm{~Hz}$, if the time interval is $1.1 \mathrm{~s}$ the virtual instrument allow no accurate frequency reading. The achieved spectrum is shown in figure $1 \mathrm{c}$ by blue lines. Obviously, correct frequency is not identified.

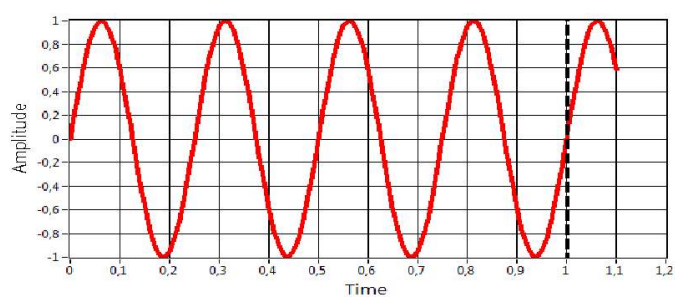

a.

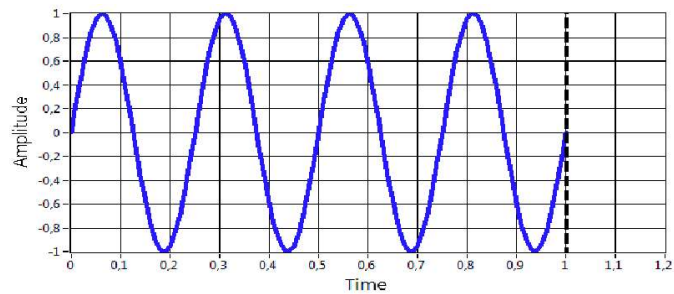

b.

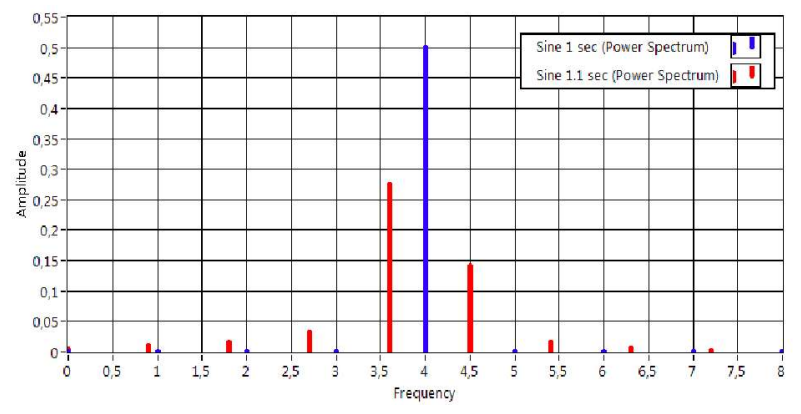

c.

Fig. 1. Two signals, one containing an integer number of periods (a) and the second with non-integer number of periods

(b) and their frequency spectra (c)

Having a look on figure 1c, we remark that although the spectral lines are denser, the frequency cannot be read accurately, while we found the peak amplitude at $3.63 \mathrm{~Hz}$ instead of $4 \mathrm{~Hz}$. Even for an improved frequency resolution, the frequency value cannot be properly read because the spectral line does not coincide with the frequency value. This has as source the phenomenon known as "leakage", which is the energy dispersion from the true frequency of the signal into several false frequencies. Because this, leakage causes an apparent amplitude decrease, while the frequency is indicated at a spectral line that does not coincide with the real one [2]. The rest of the energy is found, erroneously, on the other spectral lines.

\section{Actual methods to improve frequency identification}

Several simple methods to improve the frequency readability are known. One of the most popular is zeropadding, which consists in adding a string of zeros to the signal before the PSD is calculated [3]. It allows a better approximation to the frequencies contained in a signal. By using this method, the frequency distance between the spectral lines is decreased; therefore, an improved frequency evaluation is possible [3]. A faster algorithm than zero-padded FFT is presented in [4]. The attained results are similar with those resulted by as zero-padding, but the computational effort is essentially reduced.

Windowing is another method often used to improve frequency evaluation. For a finite-length signal that consists of a non-integer number of cycles, the application of a window smoothes its ends, making the amplitude to vary gradually towards zero at the ends [56]. This minimizes the discontinuities of the signal edges, reducing spectral leakage. However, this method works well for identification of close-located frequencies but does not improve the readability.

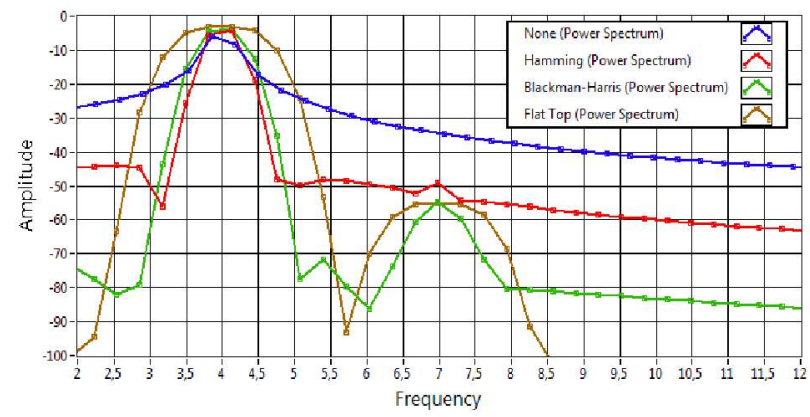

Fig. 2. The frequency spectrum for a two-tone signal in the absence and the presence of windowing - $\mathrm{dB}$ representation

It is also possible to evaluate the frequency by plotting a curve best fitting to three consecutive points, located around the estimated frequency, obtained by DFT. In figure 3 , these points are $B\left(f_{j-1}, A_{j-1}\right), C\left(f_{j}, A_{j}\right)$, $D\left(f_{j+1}, A_{j+1}\right)$, where $k-1, k$ and $k+1$ are the spectral line numbers, $f_{j-1}, f_{j}$ and $f_{j+1}$ the frequencies indicated at that lines and $A_{k-1}, A_{k}$ and $A_{k+1}$ the frequency amplitudes associated with these lines. The presumed correct frequency $f_{\text {corr }}$ and amplitude $A_{\max }$ are considered at the point in that the graph has a maximum. This peak is located on a virtual spectral line which does not exist in the calculated spectrum. It is found via a fractional correction term $\delta$, as $\mathrm{f}_{\text {corr }}=f_{j}+\delta$. There are a number of spectral peak location estimator methods for precise frequency estimation in the DFT domain [7]. In [8] parabola approximation for peak determination is used, so the correction term is given by

$\delta=\frac{a_{k+1}-a_{k-1}}{4 a_{k}-2 a_{k-1}-2 a_{k+1}}$

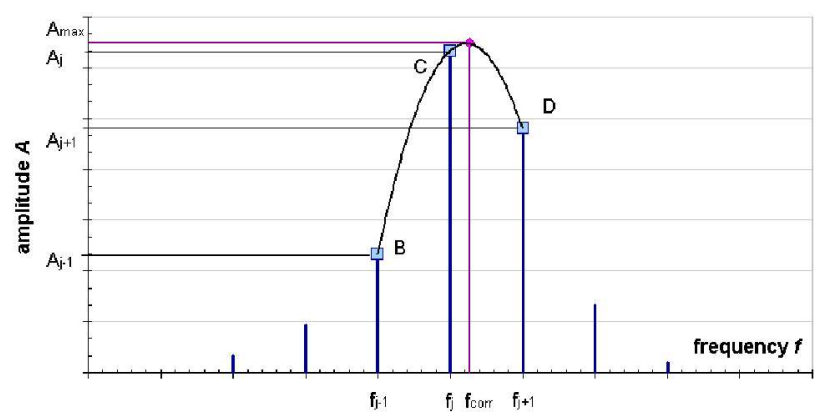

Fig. 3. The interpolation of three points from DFT

For exemplification of the method, again the sine signal of $4 \mathrm{~Hz}$ is used. The frequency resolution $\Delta f=1 / t$ 
is dependent on the signal time length $t$. Spectral analyses for different time lengths $\left(t_{\min }=0.9 \mathrm{~s}\right.$ and $t_{\max }=1.12 \mathrm{~s}$ with a step of $0.02 \mathrm{~s}$ ) are considered, resulting in different frequency resolutions. The results are indicated in table 1.

Having a look onto this table we find the amplitudes of the three points involved in the curve fitting, the fractional correction term, the values of read frequency and the values of corrected frequency obtained with the presented method. As can be seen in table 1, by using the so-called three-point method, the error between a read frequency and the corrected frequency is less than $7 \%$. In figure 4 the actual frequency, that obtained by the threepoint method and that by standard evaluation (direct reading the virtual instrument) are plotted. Observe that there are areas where the frequency values obtained by the two methods are similar (white background), while in other areas (gray background) large differences between the obtained frequencies values occur. The differences become smaller with the increase of the analysis time interval, as shown in Table 1.
From figure 4 it can be also deduced that the error is cyclical, depending on how the three considered points are placed around the real frequency.

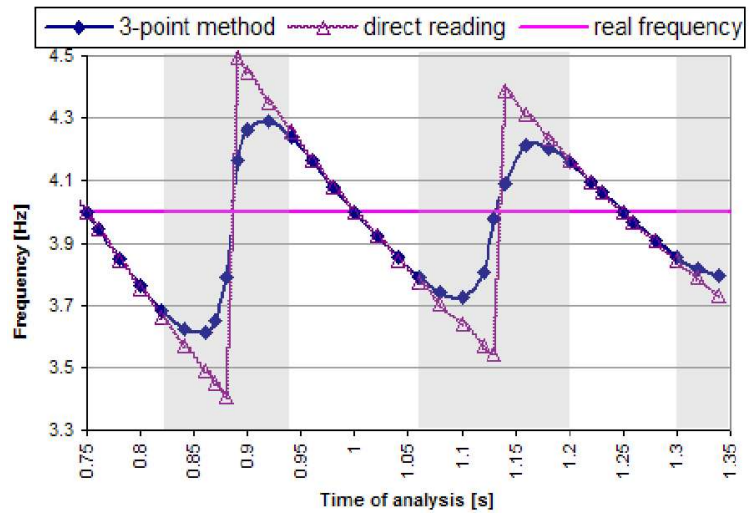

a.

Fig. 4. Comparison between the real frequency, obtained by 3points method and by direct reading (a) and the difference between the corrected and read frequency (b)

Table 1. The read frequencies and fractional correction term

\begin{tabular}{|c|c|c|c|c|c|c|c|c|}
\hline $\begin{array}{c}\mathrm{t} \\
\mathrm{s}]\end{array}$ & $\begin{array}{c}\Delta \mathrm{f} \\
{[\mathrm{Hz}]}\end{array}$ & $\begin{array}{c}\mathrm{f}_{\text {read }} \\
{[\mathrm{Hz}]}\end{array}$ & $\begin{array}{c}\mathrm{A}_{\mathrm{k}-1} \\
{\left[\mathrm{~mm} / \mathrm{s}^{2}\right]}\end{array}$ & $\begin{array}{c}\mathrm{A}_{\mathrm{k}} \\
{\left[\mathrm{mm} / \mathrm{s}^{2}\right]}\end{array}$ & $\begin{array}{c}\mathrm{A}_{\mathrm{k}+1} \\
{\left[\mathrm{~mm} / \mathrm{s}^{2}\right]}\end{array}$ & $\begin{array}{c}\delta \\
{[\mathrm{Hz}]}\end{array}$ & $\begin{array}{c}\mathrm{f}_{\text {corr }} \\
{\left[\mathrm{mm} / \mathrm{s}^{2}\right]}\end{array}$ & $\begin{array}{c}\text { Error } \\
{[\%]}\end{array}$ \\
\hline 1.12 & 0.892857 & 3.57143 & 0.03298 & 0.24430625 & 0.166872 & 0.23184 & 3.80327 & -4.91829 \\
\hline 1.1 & 0.909091 & 3.63636 & 0.031359 & 0.30906909 & 0.114683 & 0.088249 & 3.72461 & -6.8847 \\
\hline 1.08 & 0.925926 & 3.70370 & 0.024568 & 0.36470093 & 0.073694 & 0.038918 & 3.74262 & -6.43445 \\
\hline 1.06 & 0.943396 & 3.77358 & 0.015547 & 0.41093113 & 0.041821 & 0.017184 & 3.79077 & -5.23079 \\
\hline 1.04 & 0.961538 & 3.84615 & 0.007444 & 0.44977019 & 0.018447 & 0.006297 & 3.85245 & -3.68873 \\
\hline 1.02 & 0.980392 & 3.92157 & 0.002029 & 0.48110196 & 0.004398 & 0.001239 & 3.92281 & -1.92981 \\
\hline 1 & 1 & 4.00000 & 0 & 0.5 & 0 & 0 & 4.00000 & 0 \\
\hline 0.98 & 1.020408 & 4.08163 & 0.002906 & 0.49826531 & 0.003295 & 0.000196 & 4.08183 & 2.045723 \\
\hline 0.96 & 1.041667 & 4.16667 & 0.01473 & 0.46947917 & 0.010113 & -0.00253 & 4.16414 & 4.103522 \\
\hline 0.94 & 1.06383 & 4.25532 & 0.041063 & 0.41407979 & 0.016013 & -0.01624 & 4.23908 & 5.976895 \\
\hline 0.92 & 1.086957 & 4.34783 & 0.085721 & 0.34072065 & 0.018509 & -0.05822 & 4.28960 & 7.240122 \\
\hline 0.9 & 1.111111 & 4.44444 & 0.147096 & 0.26275333 & 0.017829 & -0.17925 & 4.26520 & 6.629933 \\
\hline
\end{tabular}

\section{The proposed method}

The method proposed in this paper requires an iterative time signal truncation, followed by a spectral analysis. The aim is to obtain numerous spectra with different frequency resolutions and superpose them in an "overlapped spectrum". This helps to increase the number of spectral lines in the final spectrum, as shown in figure 5 , where three spectra with different frequency resolutions: $\Delta f_{1}$ $=1 \mathrm{~Hz}, \Delta f_{2}=1.05 \mathrm{~Hz}$ and $\Delta f_{3}=1.11 \mathrm{~Hz}$ are involved.

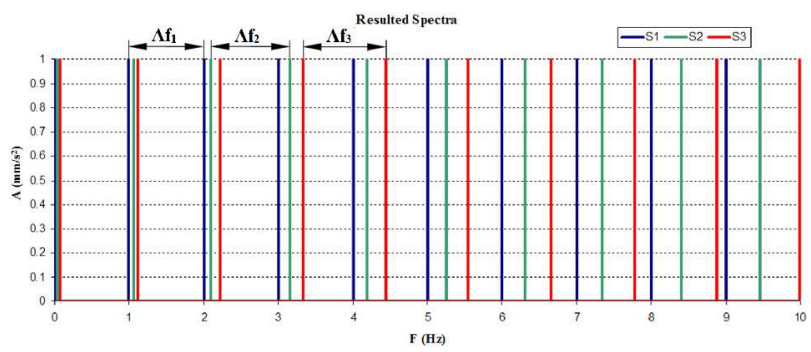

Fig. 5. Spectral lines distribution for the overlapped spectrum
From the overlapped spectrum the frequency associated with the amplitude presenting local maxima is extracted, indicating the most credible frequency value. Figure 6 exemplifies this for the frequency of $9.753 \mathrm{~Hz}$. Note that 47 iterations are performed in order to ensure a dense spectral line net.

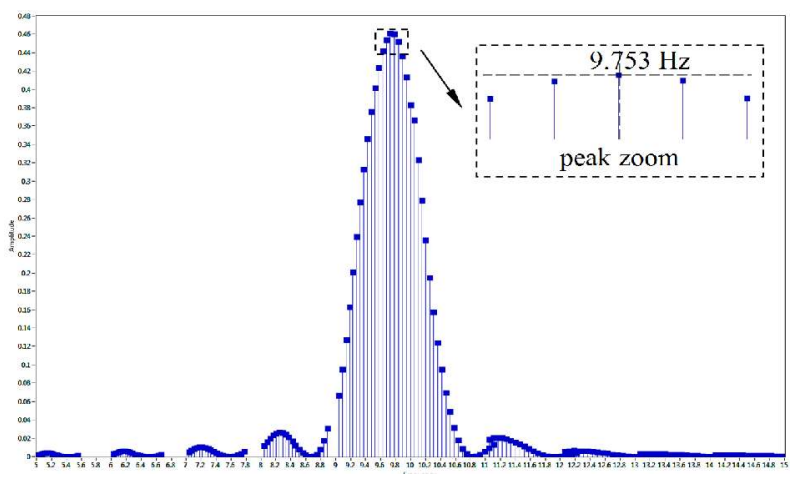

Fig. 6. The amplitude dependence on time intervals 
The process is simple and contains several steps to be performed:

- a rough evaluation of the frequencies is made using PS, by direct reading;

- the frequencies of interest for the damage detection process are identified;

- the analysis time is set for in order to ensure a frequency resolution placing a spectral line close under the presumed frequency;

- the signal length is iteratively shortened by applying a rectangular window and the PS is derived;

- the observed spectral line will migrate to superior frequencies, because the analysis time decreases;

- first an amplitude increase will be observed until a maximum is achieved, afterward the amplitudes will decrease;

- the frequency associated with the peak amplitude is extracted as the real frequency.

The time length of the vibration signal acquired for damage detection should exceed five cycles of the investigated frequency. This time was to be truncated until one period is suppressed. In this way the achievement of the amplitude peak is certain.

It should be mentioned that for each frequency an individual analysis is necessary because the time periods are different for each of them. This constitutes no impediment to the damage detection process, while the analysis for several frequencies is performed in seconds.

Fine frequency evaluation ensures reliable data for vibration-based damage detection [9, 10], making possible the observation of small modal parameter changes. Figure 7 illustrates a lab stand designed to test the sensitivity of the proposed method.

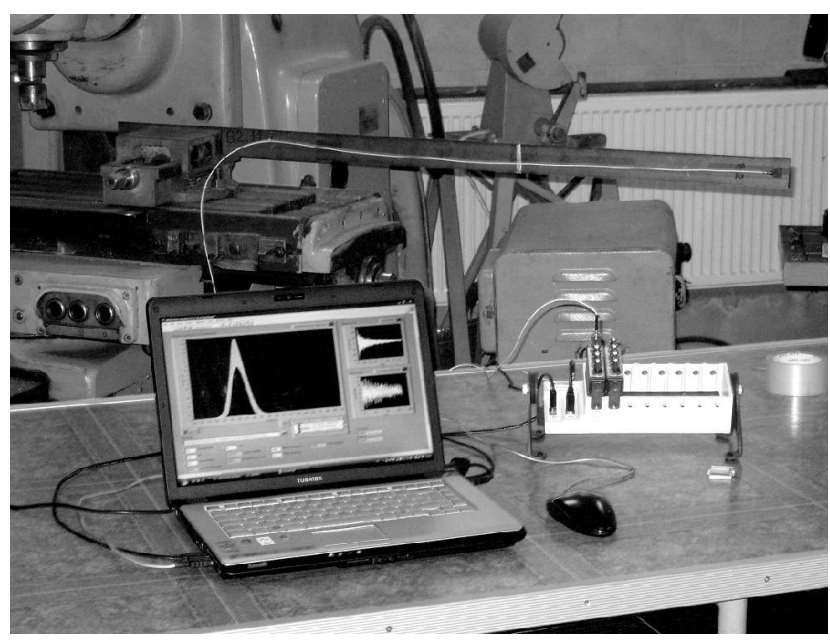

Fig. 7. Stand for testing the sensitivity of the proposed frequency evaluation method

The laptop screen shows the virtual instrument designed in LabVIEW to automatically perform the seven steps described at the beginning of this section. The two graphs at the right present the original signal and signal resulted after truncation. The upper left graph shows the overlapped spectrum which has a clear peak. In addition, the frequency and amplitude values associated to this peak are numerically indicated.

\section{Conclusion}

In this paper, a simple method for an accurate frequency evaluation is proposed and compared with actual methods presented in the literature. Even if these methods improve the frequency readability, they are not enough accurate to evaluate the changes occurring in structures due to small damages. The proposed method bases on overlapping numerous spectra achieved from the same signal analysed for different time lengths. By overlapping, the number of spectral lines in the frequency domain of interest is dramatically increased, thus results the possibility to identify the peak amplitude and consequently the frequency with high precision. Test performed to reveal the frequency sensitivity shown that the frequency is identified with a precision of three digits after unit.

\section{Acknowledgement}

The work has been funded by the Sectoral Operational Programme Human Resources Development 2007-2013 of the Ministry of European Funds through the Financial Agreement POSDRU/159/1.5/S/132395.

\section{References}

1. Z. Praisach, G.R. Gillich, D.E. Birdeanu, Considerations on Natural Frequency Changes in Damaged Cantilever Beams Using FEM, Latest Trends on Engineering Mechanics, Structures, Engineering Geology (2010)

2. S.T. Abed, Z.E. Dallalbashi, F.A. Taha, Studying the Effect of Window Type on Power Spectrum Based on MatLab, Tikrit J. Eng. Sciences 19(2) (2012)

3. S.A. Neild, Using nonlinear vibration techniques to detect damage in concrete bridges, $\mathrm{PhD}$ thesis, Univ. of Oxford, Oxford, U.K (2001)

4. C. Donciu, M. Temneanu, An alternative method to zero-padded DFT, Measurement 70 (2015)

5. G. Andria, M. Savino, A. Trotta, Windows and interpolation algorithms to improve electrical measurement accuracy, IEEE Transactions on Instrumentation and Measurement, 38(4) (1989)

6. S.T. Abed, Z.E. Dallalbashi, F.A. Taha, Studying the Effect of Window Type on Power Spectrum Based on MatLab, Tikrit J. Eng. Sciences 19(2) (2012)

7. Ç. Candan, A Method For Fine Resolution Frequency Estimation From Three DFT Samples, IEEE Signal Processing Letters, 18(6) (2011)

8. P. Voglewede, Parabola approximation for peak determination, Global DSP Mag., 3(5), (2004)

9. G.R. Gillich, Z.I. Praisach, M.A. Wahab, O. Vasile, Localization of Transversal Cracks in Sandwich Beams and Evaluation of Their Severity, Shock Vib. Article Number: 607125 (2014)

10. G.R. Gillich, Z.I. Praisach, Modal identification and damage detection in beam-like structures using the power spectrum and time-frequency analysis, Signal Process. 96 (2014) 